\title{
COVID-19: Stroke Admissions, Emergency Department Visits, and Prevention Clinic Referrals
}

\author{
Maria Bres Bullrich, Sebastian Fridman (1), Jennifer L. Mandzia, Lauren M. Mai, \\ Alexander Khaw, Juan Camilo Vargas Gonzalez (iD, Rodrigo Bagur, Luciano A. \\ Sposato
}

\begin{abstract}
We assessed the impact of the coronavirus disease 19 (COVID-19) pandemic on code stroke activations in the emergency department, stroke unit admissions, and referrals to the stroke prevention clinic at London's regional stroke center, serving a population of 1.8 million in Ontario, Canada. We found a 20\% drop in the number of code strokes in 2020 compared to 2019, immediately after the first cases of COVID-19 were officially confirmed. There were no changes in the number of stroke admissions and there was a $22 \%$ decrease in the number of clinic referrals, only after the provincial lockdown. Our findings suggest that the decrease in code strokes was mainly driven by patient-related factors such as fear to be exposed to the SARS-CoV-2, while the reduction in clinic referrals was largely explained by hospital policies and the Government lockdown.

RÉSUMÉ : Hospitalisations à la suite d'un AVC, visites à un service des urgences et aiguillage vers une clinique de prévention des AVC dans le contexte de la pandémie de coronavirus (COVID-19). Nous avons tenté d'évaluer l'impact de la pandémie de coronavirus (COVID-19) sur l'activation de codes d'alerte correspondant aux AVC dans un service des urgences mais aussi sur la prise en charge de patients dans une unité de soins de l'AVC et sur leur aiguillage vers une clinique de prévention des AVC située dans le centre régional de traitement des AVC de London en Ontario. À noter que cet établissement dessert une population d'environ 1,8 million d'habitants. Immédiatement après que les premiers cas de coronavirus (COVID-19) eurent été officiellement confirmés, nous avons constaté en 2020 une baisse de $20 \%$ du codage en lien avec des AVC par rapport à l'année 2019. Aucun changement n'a été par ailleurs noté en ce qui concerne le nombre d'hospitalisations consécutives à des AVC ; cela dit, une diminution de $22 \%$ a été observée en ce qui regarde le nombre de cas adressés à des spécialistes, et ce, seulement après la mise en place de mesures de confinement dans la province. Nos résultats suggèrent donc que la diminution du codage en lien avec des AVC peut s'expliquer principalement par des facteurs liés aux patients eux-mêmes, par exemple la crainte d'être exposé au coronavirus, tandis que la diminution du nombre de cas adressés à des spécialistes peut s'expliquer en grande partie par les politiques de ce centre hospitalier et les mesures de confinement des autorités responsables.
\end{abstract}

Keywords: STROKE, COVID-19, prevention

doi:10.1017/cjn.2020.101

Can J Neurol Sci. 2020; 47: 693-696

There is a growing concern that patients with stroke symptoms are not seeking medical attention in the emergency department (ED) because of fear of being exposed to the severe acute respiratory syndrome coronavirus-2 (SARS-CoV-2). ${ }^{1,2}$ However, to date, no study has formally evaluated the impact of the coronavirus disease 19 (COVID-19) pandemic on the number of stroke patients being assessed in the ED, stroke admissions, or referrals to stroke prevention clinics in Canada. We, therefore, assessed the impact of the coronavirus disease 19 (COVID-19) pandemic on code stroke activations in the ED, stroke unit admissions, and referrals to the urgent stroke prevention clinic at London Health Sciences Center University Hospital's regional stroke center, serving a captive population of 1.8 million in Ontario, Canada.

The primary outcome of this study was the number of code strokes. Secondary outcomes were stroke unit admissions and urgent stroke prevention clinic referrals. The exposure was the COVID-19 pandemic. We quantified the number of code strokes in 12 World Health Organization (WHO) epidemiological weeks between February 3, 2020, and April 26, 2020, by interrogating the hospital's wireless paging system database. We chose February 3, 2020, as the start date of the study period because it was the first Monday after the COVID-19 case was confirmed in London on Friday January 31, 2020. This was the third confirmed case in Ontario. The study period started on a Monday

From the Department of Clinical Neurological Sciences, Schulich School of Medicine and Dentistry, Western University, London, Ontario, Canada (MBB, SF, JLM, LMM, AK, JCVG, LAS); Division of Cardiology, Department of Medicine, Schulich School of Medicine and Dentistry, Western University, London, Ontario, Canada (RB); Department of Epidemiology and Biostatistics, Schulich School of Medicine and Dentistry, Western University, London, Ontario, Canada (RB, LAS); Heart \& Brain Laboratory, Western University, London, Ontario, Canada (LAS); Department of Anatomy and Cell Biology, Schulich School of Medicine and Dentistry, Western University, London, Ontario, Canada (LAS); Lawson Health Research Institute, London, Ontario, Canada (LAS); and Robarts Research Institute, Western University, London, Ontario, Canada (LAS)

Received May 14, 2020. Final Revisions Submitted May 19, 2020. Date of Acceptance May 19, 2020.

Correspondence to: Luciano A. Sposato, 339 Windermere Rd., Rm A10-322, London, ON, Canada, N6A 5A5. Email: Luciano.Sposato@LHSC.on.ca 
A

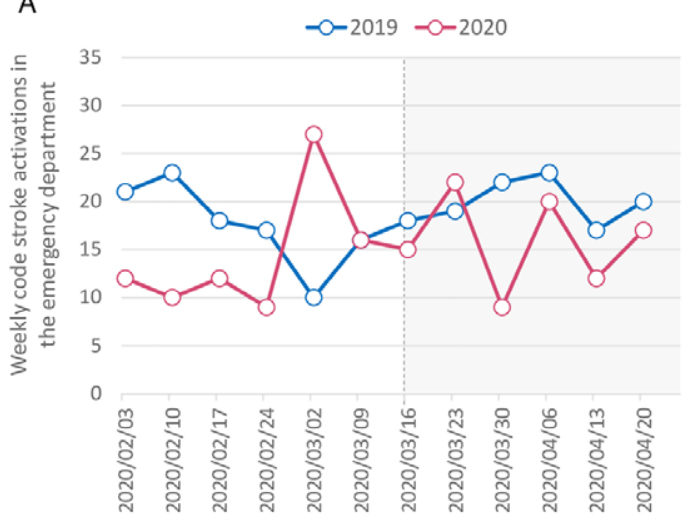

C

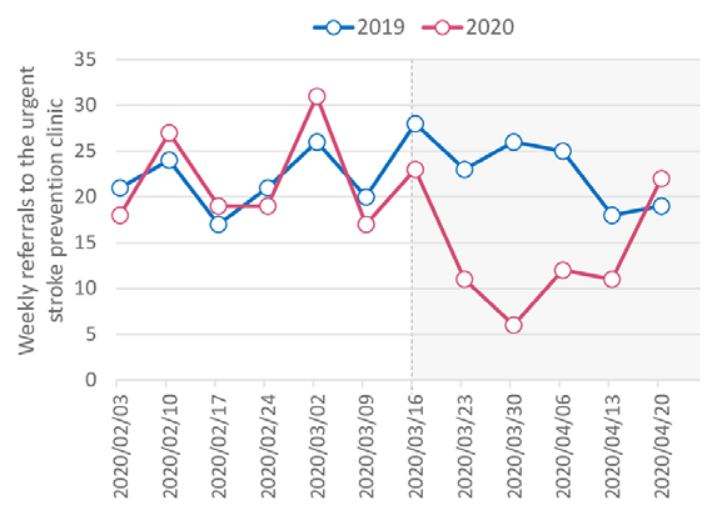

B

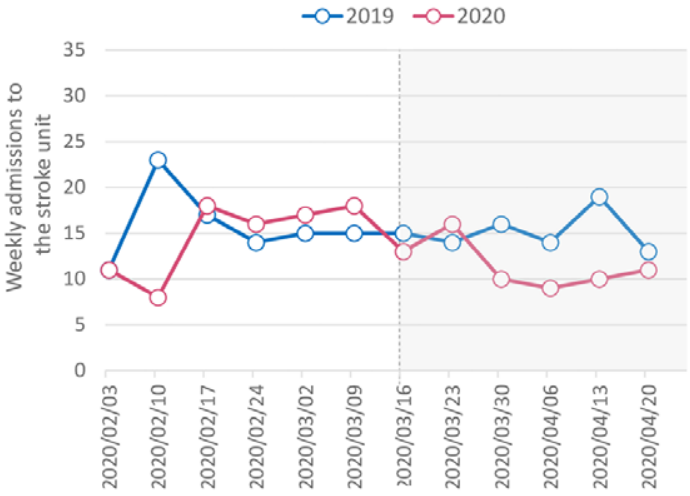

D

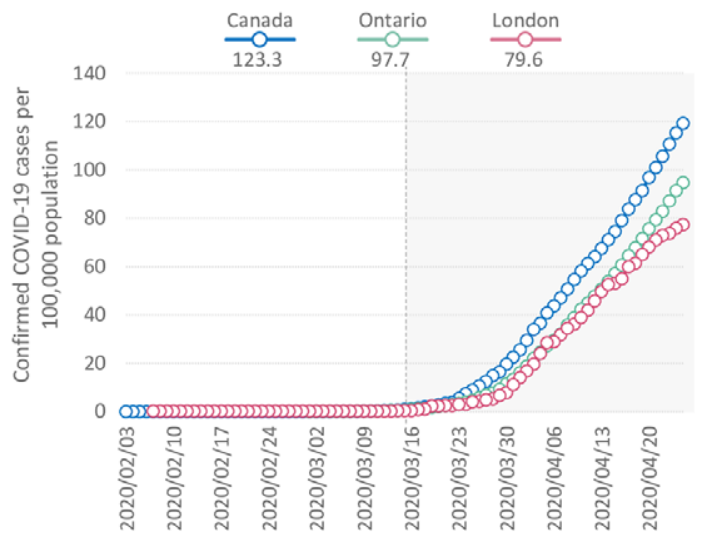

Figure 1: Weekly stroke team activations and urgent stroke prevention clinic referrals. Panel A: Weekly code stroke activations in the ED. Panel B: Weekly stroke admissions. Panel C: Weekly referrals to the urgent stroke prevention clinic. Panel D: Confirmed cases of COVID-19 in London, Ontario, and Canada per 100,000 population since February 3, 2020. The vertical line represents the date of the provincial lockdown and the shades show the time window thereafter.

because this is the first day of WHO epidemiological weeks and it also allows for mitigating the bias of weekdays vs. weekend consults. We used the same WHO epidemiological weeks of 2019 as a comparator. We compared epidemiological weeks of 2020 vs. 2019. These are matched weeks in both years, which are used for mitigating the bias of seasonal variations in stroke consults. Code strokes included patients brought to the ED by the Emergency Medical Services and walk-in strokes. Additionally, we quantified hemorrhagic and ischemic stroke admissions to the stroke unit and referrals to the urgent stroke prevention clinic during the same period by reviewing the London Ontario Stroke Registry, the inpatient census, and London Health Sciences Centre's electronic booking system. The stroke registry is updated on a real-time basis. Clinic referrals consist of patients with minor strokes or transient ischemic attacks (TIAs).

On March 16, 2020, the Government of Ontario implemented a provincial lockdown advising Ontarians to avoid gatherings of over 50 people, and the closure of recreational libraries, private schools (public schools were closed on March 12), daycares, churches and other faith settings, and all bars and restaurants. On the same day, after a provincial order requesting all hospitals to implement pandemic plans by ramping down nonemergent clinical activities, the hospital implemented a service reduction policy. This resulted in the immediate transition of most outpatient clinics to telemedicine systems. On March 23, 2020, the Provincial Government expanded the lockdown by ordering the mandatory closure of all nonessential workplaces and the enhancement of social distancing.

To compare code strokes, stroke unit admissions, and clinic referrals in 2020 against 2019, we plotted weekly numbers of consults in both years. We also plotted the number of confirmed COVID-19 cases in London, Ontario, and Canada per 100,000 population as a reference of the local progression of the pandemic.

The study data comprised a count of cases over a unit of time (epidemiological weeks), which follows a Poisson distribution. For this reason, we decided to use a Poisson model. Due to the possibility of overdispersion of the data, we compared the results of negative binomial models and the Poisson regressions for all outcomes by using the maximum likelihood method. In case of no differences between both types of models, we opted for the parsimonious Poisson model. The number of code strokes, stroke unit admissions, and urgent stroke prevention clinic referrals were the outcome of interest in their respective models. We designed the models to test if there were effects attributable to the year (2019 vs. 2020) as a surrogate of the COVID-19 pandemic or to the provincial lockdown on the weekly number of cases. 
The number of code strokes, stroke unit admissions, and stroke prevention clinic referrals were 224,186 , and 268 in 2019 vs. 181, 157, and 216 in 2020 (Figure 1). Data followed a Poisson distribution except for stroke prevention clinic referrals in 2020. We therefore developed a negative binomial model. We found no differences between the Poisson and negative binomial model, so we retained the former for all the prespecified outcomes.

The model for code strokes revealed that there was a significant 23\% reduction in the overall number of events in 2020 relative to 2019 (95\%CI 2.0\%-30.4\%). However, the March 16 provincial lockdown was not significantly associated with any further decrease in the number of consults. Accordingly, the figure shows that most of the reduction in code strokes occurred during the 4 weeks of the pandemic preceding the provincial lockdown.

In the stroke unit admission model, there were no significant differences between 2019 and 2020, either before or after the provincial lockdown.

The model for urgent stroke prevention clinic referrals showed no significant changes between 2019 and 2020 before the landmark date of March 16. However, there was a significant decrease of $35.2 \%$ (95\% CI $24.9 \%-51.8 \%$ ) in the number of referrals after the provincial lockdown. The figure illustrates steepest reduction in the number of clinic referrals was observed after the provincial lockdown and the consequent implementation of the hospital's service reduction policy.

Our findings offer insight into stroke systems of care utilization during the 2020 COVID-19 pandemic in London, Ontario, Canada. Code strokes dropped during the first 4 weeks of February 2020, corresponding to the period after the first confirmed COVID-19 cases were officially reported in the province. There was no association between the provincial lockdown and the decrease in code strokes. As such, in line with worldwide concerns, we hypothesized that patients' fears to be exposed to the SARS-CoV-2 in the ED as soon as the first COVID-19 cases were officially confirmed in London and the province may have influenced their decision to stay at home instead of seeking medical attention.

Interestingly, despite a reduction in the number of code strokes, we did not find significant differences in the number of admissions, which remain relatively stable during 2020 . This is conceivably explained by a pandemic-related selection of patients attending the ED, resulting in fewer stroke mimics and a larger proportion being admitted. It is also possible that patients with TIAs or very mild strokes (so that they do not qualify for either code strokes or for admission) decided to stay at home and not seek medical attention. These potential explanations remain on hypothetical grounds and warrant further investigation.

The number of urgent stroke prevention clinic referrals did not change significantly during the first 4 weeks of February 2020. Yet, we observed a steep drop after March 16, 2020, meaning that the provincial lockdown may have impacted on physicians' willingness or ability to refer patients. The hospital's transition into a telemedicine system and a perceived uncertainty about whether the clinic was processing referrals may have also played a role.

A recent report showed a drop in the numbers of patients in a commercial neuroimaging database from the USA associated with the RAPID software platform (iSchemaView). However, this study used a surrogate of the number of patients being assessed for acute ischemic stroke. ${ }^{3}$ Another small study from the USA showed a drop in the number of stroke admissions, transfers from referral centers, telemedicine consultations, and ED visits. ${ }^{4}$ However, this study did not account for seasonal changes in stroke consults.

In summary, these concerning results should encourage Governments, scientific societies, and other relevant stakeholders to take urgent action aiming at improving stroke patients' awareness about the importance of seeking medical attention when experiencing stroke symptoms. Health systems will also need to be prepared for future pandemics and for the backlash caused by fewer cerebrovascular events being prevented in patients with minor strokes or TIAs who will not receive the appropriate preventive treatments during the COVID-19 pandemic because of not reporting their symptoms. ${ }^{5}$

\section{ACKNowledgements}

We specially thank Naomi Reintjes for collaborating with the stroke unit census.

\section{Funding}

LAS is supported by the Kathleen \& Dr. Henry Barnett Research Chair in Stroke Research (Western University, London, Ontario, Canada) and the Edward and Alma Saraydar Neurosciences Fund (London Health Sciences Foundation).

\section{Disclosures}

None of the authors have significant disclosures related to this work.

\section{STATEMENT OF AUTHORSHIP}

MBB contributed to drafting the first manuscript and reviewed the final version of the manuscript for intellectual content.

SF planned and performed the statistical analysis, and reviewed the final version of the manuscript for intellectual content.

JLM reviewed the final version of the manuscript for intellectual content.

LMM reviewed the final version of the manuscript for intellectual content.

AK reviewed the final version of the manuscript for intellectual content.

JCVG planned and performed the statistical analysis, and reviewed the final version of the manuscript for intellectual content.

RB conceived the study, planned the statistical analysis, and reviewed the final version of the manuscript for intellectual content.

LAS conceived and supervised the study, planned the statistical analysis, gathered the data, drafted the first manuscript, and reviewed the final version of the manuscript for intellectual content.

\section{REFERENCES}

1. Sheth K. Hospital admissions for strokes appear to have plummeted, a doctor says, a possible sign people are afraid to seek critical help. Washington Podt; 2020. https://www.washingtonpost.com/national/ health-science/hospital-admissions-for-strokes-appear-to-haveplummeted-a-doctors-says-a-possible-sign-people-are-afraid-toseek-critical-help/2020/04/08/2048b886-79ac-11ea-b6ff-597f 170df8f8_story.html; Accessed April 22, 2020. 
2. Brainin M. Stroke Care and the COVID19 Pandemic. WSO News \& Blog; 2020. https://www.world-stroke.org/news-and-blog/news/ stroke-care-and-the-covid19-pandemic; Accessed on April 22, 2020.

3. Kansagra AP, Goyal MS, Hamilton S, Albers GW. Collateral Effect of Covid-19 on Stroke Evaluation in the United States. N Engl J Med. 2020:NEJMc2014816. doi: 10.1056/NEJMc2014816. Online ahead of print.
4. Siegler JE, Heslin ME, Thau L, Jovin TG. Falling stroke rates during COVID-19 pandemic at a Comprehensive Stroke Center. Stroke Cerebrovasc Dis. 2020;29(8):104953. doi: 10.1016/j.jstroke cerebrovasdis.2020.104953. Online ahead of print.

5. Lavallée PC, Meseguer E, Abboud H, et al. A transient ischaemic attack clinic with round-the-clock access (SOS-TIA): feasibility and effects. Lancet Neurol. 2007;6:953-960. 